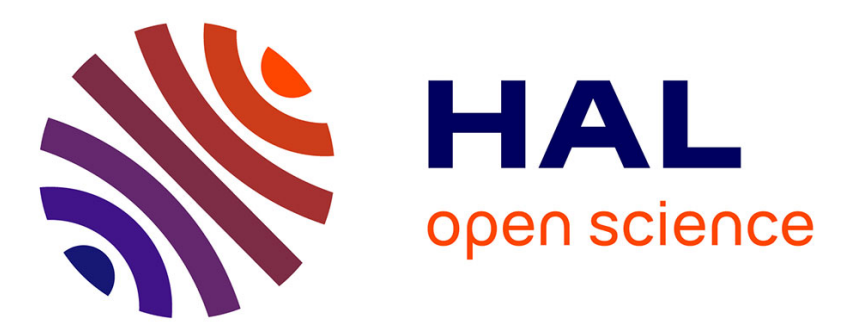

\title{
Crop-Rotation Structured Classification using Multi-Source Sentinel Images and LPIS for Crop Type Mapping
}

\author{
S. Bailly, Sébastien Giordano, Loic Landrieu, N. Chehata
}

\section{- To cite this version:}

S. Bailly, Sébastien Giordano, Loic Landrieu, N. Chehata. Crop-Rotation Structured Classification using Multi-Source Sentinel Images and LPIS for Crop Type Mapping. IGARSS 2018 - 2018 IEEE International Geoscience and Remote Sensing Symposium, Jul 2018, Valencia, France. pp.1950-1953, 10.1109/IGARSS.2018.8518427 . hal-02387132

\section{HAL Id: hal-02387132 \\ https://hal.science/hal-02387132}

Submitted on 29 Nov 2019

HAL is a multi-disciplinary open access archive for the deposit and dissemination of scientific research documents, whether they are published or not. The documents may come from teaching and research institutions in France or abroad, or from public or private research centers.
L'archive ouverte pluridisciplinaire HAL, est destinée au dépôt et à la diffusion de documents scientifiques de niveau recherche, publiés ou non, émanant des établissements d'enseignement et de recherche français ou étrangers, des laboratoires publics ou privés. 


\title{
CROP-ROTATION STRUCTURED CLASSIFICATION USING MULTI-SOURCE SENTINEL IMAGES AND LPIS FOR CROP TYPE MAPPING
}

\author{
S. Bailly ${ }^{1}$, S. Giordano ${ }^{1}$, L. Landrieu ${ }^{1}$, N. Chehata ${ }^{1,2}$ \\ 1. Univ. Paris-Est, LASTIG MATIS, IGN, ENSG, F-94160 Saint-Mande, France \\ 2 : EA G\&E Bordeaux INP / Université Bordeaux Montaigne, France
}

\begin{abstract}
Automatic analysis of Sentinel image time series is recommended for monitoring agricultural land use in Europe. To improve classification capacities, we propose a temporal structured classification combining Sentinel images and former vintages of the Land-Parcel Identification System. Inter-annual crop rotations are learned and combined with the satellite images using a Conditional Random Field. The proposed methodology is tested on a $233 \mathrm{~km}^{2}$ study area located in France and with a 25 categories national nomenclature. The classification results are globally improved.
\end{abstract}

Index Terms - Classification, Temporal regularization, Conditional Random Fields, Agriculture, Monitoring, Sentinel.

\section{INTRODUCTION}

Sentinel image time series exhibit unprecedented characteristics that are perfectly tailored with agriculture monitoring. Spatial resolution of both optical and radar images are very consistent with a parcel-level approach. More remarkably, the high temporal frequency of acquisition (5-6 days) and the fact that this program is at least maintained until 2030 pave the way to chronicle short and long-term evolutions. In Europe, several use-cases for agricultural monitoring with Sentinel images have been proposed [1] such as for example crop monitoring, controlling Common Agricultural Policy (CAP) payments with remote sensing, updating and quality control of the Land Parcel Identification System (LPIS) or precision farming at the farm-level. In the LPIS, the information on the agricultural parcels (cover types and areas) are usually derived from annual declaration by the farmers followed by manual controls by national paying agencies.

In this context, recent studies have assessed the classification accuracy that can be obtained with Sentinel images on agricultural land covers [2, 3, 4, 5]. However, in all of them the nomenclature is restricted to few and most frequent annual crops. Classes known to be challenging to determine with remote sensing such as permanent grassland or permanent crops are not taken into account. Furthermore when a parcel-based approach is used, small parcels are usually ignored $(<250$ ha in [4]).

To overcome this issue, we believe that modelling the spatio-temporal structures concerning agricultural parcels in combination with the Sentinel image time series, can lead to significant gains in accuracy with more exhaustive nomenclatures. These structures are of different types and can concern for example (i) the choice of a crop type on a given parcel (annual or multi-annual crop rotations), (ii) local similarities in events (ploughing, seeding or harvesting dates) or (iii) management practices for the parcels belonging to a same farmer. In this paper, we investigate the modelling and the integration in the classification of inter-annual crop rotation information.

Two different approaches can be used to model rotations. The first one consists in using a priori agronomist expert knowledge. Several models have been proposed and assessed [6]. However, the models are strongly dependent on the study area and have no adaptation capacity to environmental or agricultural management changes. The second approach is learning rotations as way of overcoming the limitations driven by the expert knowledge a priori approaches. The data that can be used for learning are the former versions of LPIS. This method has recently been studied by [7]. Nevertheless, only very few studies have focused on the integration of crop rotation information into classification pipelines. A prediction model based on the Markov logic is proposed by [7]. However, there is no combination with remote sensing observations.

The objective of this paper is then to propose a method to integrate the crop rotation temporal structure into a Sentinelbased classification process and to assess the capacity of the method to improve classification results. The methodology is explained in Section 2, the experimental set-up (study area and datasets) along with result analysis are proposed in Section 3. Conclusions are drawn in Section 4.

\section{METHODOLOGY}

The classification method proposed is made up with a data term (Section 2.1 combined with a Conditional Random Fields (CRF) for temporal structured prediction (Section 2.2). 


\subsection{Parcel-based multi-source and multi-temporal clas- sification}

The data term is obtained with common state-of-the art methods. Sentinel images were automatically downloaded from the French national mirror Peps [8] (Sentinel-1) in the Ground Range Detected (GRD) format and from the national downstream service Theia [9] (Sentinel-2) in ground geometry and calibrated in Top of Canopy (TOC) reflectance. Dual polarisation GRD Sentinel-1 images were first calibrated to $\sigma_{0}$ radar backscattering coefficient. Orthorectification is performed using the SRTM digital terrain model and the georeferencing information supplied with the GRD files. The speckle is slightly removed using a simple $5 \times 5$ Lee filter [10]. An extra radar feature $\left(\frac{\sigma_{0_{V H}}}{\sigma_{0_{V V}}}\right)$ is computed. This ratio is known as being more independent to the slope and moisture content effects of each agricultural parcel [11]. For optical images, the missing data (clouds) are filled in using provided cloud masks with a multi-temporal spline interpolation. Average and standard deviation of these 3 radar channels and the 10 optical reflectance bands are then computed for each date of the satellite time series for each agricultural parcel geometry of the LPIS. At the parcel-based level, a Random Forest classifier is used. Decision tree votes are gathered to model the data term probabilities $p\left(y_{n} \mid x_{n}\right)$ of Equation 2, $y_{n}$ being the agricultural classes considered and $x_{n}$ the parcel-based multi-source and multitemporal time serie features.

\subsection{Temporal-structured classification of parcels}

In order to model the temporal structure of the crop rotations, we used the graphical model given in Figure 1. This first order Markov chain can be generalized to greater orders. However, because of the specifications of LPIS data, only the first order has been applied and assessed in this paper. Indeed, the LPIS are known at the parcel-block level (i.e. a group of contiguous parcels with the same operator but possibly different land covers) before 2015. The agricultural parcel class of a given $n$ year is modeled by a random variable $Y_{n}$. We consider this variable only influenced by the previous year variable $Y_{n-1}$. The satellite observations of each $n$ year $X_{n}$ are taken into account with a Conditional Random Field (CRF).

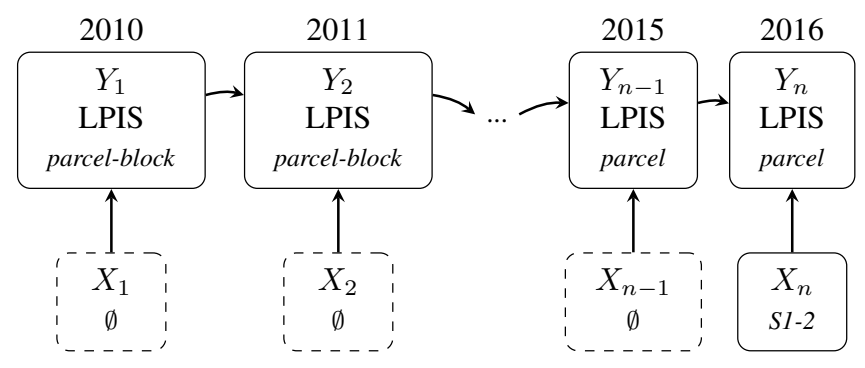

Fig. 1. Conditional Random Field.

With such a modelling and under the hypothesis of the independance between transitions $Y_{n}$ and observations $X_{n}$, the factorisation of the graphical model given in Figure 1 can be written with Equation 2

$$
p_{\theta}(\mathbf{y} \mid \mathbf{x}) \propto p\left(y_{1}\right) \times p\left(y_{1} \mid x_{1}\right) \times \prod_{i=2}^{n}\left(p\left(y_{i} \mid y_{i-1}\right) \times p\left(y_{i} \mid x_{i}\right)\right)
$$

Nevertheless, the use of $X=\left\{X_{1}, X_{2}, \ldots, X_{n-1}\right\}$ in dashed line in Figure 1 is not necessary. The former LPIS versions give a robust knowledge of the realization of the random variables $Y=\left\{Y_{1}, Y_{2}, \ldots, Y_{n-1}\right\}$. Indeed, errors concerning crop types in the initial declaration by farmers are in minority and furthermore this information is thereafter controlled by National Paying Agencies. Therefore, we propose a simpler form given in Equation 2 The data term is estimated with the Random Forest method (Section 2.1) and a temporal structure term $p\left(y_{n} \mid y_{n-1}\right)$ is necessary.

$$
p_{\theta}\left(y_{n} \mid x_{n}, y_{1}, \ldots, y_{n-1}\right) \propto \underbrace{p\left(y_{n} \mid y_{n-1}\right)}_{\text {temporal structure }} \times \underbrace{p\left(y_{n} \mid x_{n}\right)}_{\text {data term }}
$$

To estimate the regularization term $p\left(y_{n} \mid y_{n-1}\right)$, we learn a transition matrix $\widehat{M}$ built up with a series of parcel-block LPIS. An hypothesis of spatio-temporal homogeneity of the parcels is considered. Only geometrically stable and pure in terms of land cover parcel-blocks are used. Equation 3 shows how to estimate the $p\left(y_{n} \mid y_{n-1}\right)$ with past agricultural land cover transitions.

$$
\begin{aligned}
& \qquad \widehat{M_{k, l}}=\frac{n_{k, l}}{n_{k}} \\
& \text { with } n_{k, l} \text { the number of transitions } k \rightarrow l \text { (for all parcels of all } \\
& \text { years considered) and } n_{k} \text { the number of occurences of the } \\
& \text { agricultural class } k \text { (for all the parcels of the n-1 first years) }
\end{aligned}
$$

\section{RESULTS}

\subsection{Experimental Set-up}

The method has been tested on a $233 \mathrm{~km}^{2}$ study area located $60 \mathrm{~km}$ at the East of Paris. A national 25 classes land cover classification was used to estimate the transition matrix of Equation 3 on a French department administrative area (5 915 $\mathrm{km}^{2}$ ). 36891 geometrically stable and pure in terms of land covers parcel blocks contribute to the estimation of the $\widehat{M}$ matrix.

As for the data term, only the land cover classes present on the study area were learned. This agricultural classes and the number parcels considered are Corn (350 parcels), Barley (158), Other Cereals (889), Rape seed (85), Protein (peas) (76), Fiber plants (76), Forage crops (46), Permanent grassland (725), Fruit trees (30), Vegetables (131). These classes are categories and can possibly gather a large number of agricultural land covers (16 different classes for Other Cereals) on the department area. The average area of the parcels on the 
study area is 4.5 ha with high standard deviation ( $\sigma=6.5 \mathrm{ha}$ ). The 2016 parcel-based LPIS has been used for training and validation of the data term.

Table 1 gives the number of features used for the estimation of the data term. Sentinel-2 acquisition technical problems on the particular orbit covering the study area had limited the amount of optical images available.

\begin{tabular}{ccc}
\hline & Sentinel-1 (radar) & Sentinel-2 (optical) \\
\hline Number of dates & 85 & 12 \\
Features per date & 6 & 20 \\
Total & 509 & 240 \\
\hline
\end{tabular}

Table 1. Features used for the estimation of the data term.

In the following a configuration named unstructured corresponds to the results of the parcel-based classification. The Random Forest was trained with 100 decision trees using all aggregated attributes from Table 1 and a maximum depth of the trees of 15 . Then a second configuration called structured in the rest of the paper corresponds the product of the two probabilities defined in Equation 2 for classification. The 2015 parcel-based LPIS was needed to determine the necessary previous land cover observed on a parcel. 5 years (20102014) of the parcel-block LPIS were used for the estimation of the transition matrix. For the unstructured and temporal structured classification, $50 \%$ of the parcels were used for training and $50 \%$ for validation, and the results proposed are the average of 10 iterations.

The classification was assessed with global metrics (overall accuracy (OA), weighted ( $F$-score-w) and unweighted $F$ score by the number of samples of each classes) and per-class metrics (F-score, recall and precision).

\subsection{Result analysis}

The transition matrix estimated contains very relevant information. For instance, strong inter-annual crop rotation are observed. The probability to grow Other Cerals (common wheat) after Rape seed is $97.09 \%$ or Protein (peas) to Other Cereals $(94.85 \%)$. The permanent grassland remains stable (94.45\%), such as permanent crops like Fruit trees $(83.65 \%)$.

Table 2 shows the global results after 10 iterations. The results are proposed only with the radar features or the optical features (Table 1) or with their aggregation. Taking into account the temporal structure improves all the global metrics for all the feature configurations. In $O A$, we observe a gain of $+2.7 \%$ for the radar configuration, $+4.6 \%$ for the optical and $+2.6 \%$ for the combined optical and radar one).

However the improvement of classification accuracy is not homogeneous for the different agricultural classes. Table 3 evaluates the effect of taking into account the temporal structure on each class, with the optical and radar features.

First, we can notice that there is a strong improvement on the stable over time agricultural classes such as Perma-
Table 2. Global classification metrics after 10 iterations.

\begin{tabular}{c|ccc}
\hline \multicolumn{4}{|c}{ Unstructured } \\
\hline Configuration & Overall & Unweighted & Weighted \\
& Accuracy & F-score & F-score \\
& $O A$ & $F$-score & F-score-w \\
\hline Radar & 0.892 & 0.734 & 0.878 \\
Optical & 0.824 & 0.624 & 0.809 \\
Radar+Optical & 0.890 & 0.744 & 0.885 \\
\hline \multicolumn{4}{c}{ Structured } \\
\hline Radar & 0.919 & 0.776 & 0.911 \\
Optical & 0.870 & 0.675 & 0.853 \\
Radar+Optical & 0.916 & 0.762 & 0.906 \\
\hline
\end{tabular}

nent grasslands $(+8.7 \%$ in F_score $)$ and Fruit trees $(+93 \%$ in F_score). Almost all the Fruit trees validation samples were classified as Permanent grasslands in the unstructured configuration. The temporal structure is also useful to separate classes that have similar data terms. This is for example the cases for Forage crops $(+70.5 \%$ in F_score) and Permanent grasslands. These two classes are very similar in the optical and radar features. As a result, Forage crops are almost for the most part classified as permanent grasslands. The temporal structure enables to make the distinction.

Then, for annual crops that are used with regular rotation patterns, the class metrics are improved. (Protein (peas) $(+0.4 \%)$, Rape seed $(+1 \%)$, Other Cereals $(+0.7 \%)$. However, when the transitions are less respected by farmers, the temporal structure can cause a loss in the classification accuracy. This is the case for Corn $(-6.3 \%)$ or Barley $(-8.2 \%)$. For the Barley class, the decrease (Recall) is partially due to the misclassification of the Fiber Plants class. For Corn and Barley the decrease could be explained by the fact that these crops are used in 3-years rotation, a structure that the 1st-oder Markov chain can not capture completely.

Finally, there is a major drawback with the Fiber plants class (-97.4\% in F_score). For that particular case, the data term is reliable ( $97.4 \%$ in F-score) and after temporal regularization almost all the Fiber plants are classified as Barley. The result could be explained by the fact that there is a very small amount of geometrically stable Fiber plants on the area. The strong general transition rule learned for the Fiber plants could not be useful for those specific parcels.

\section{CONCLUSION}

In conclusion, we proposed a framework to take into account the crop rotation temporal structure in the classification. The classification is globally improved. The method is based on learning both data term and temporal structure. As a result, it can be easily applied to other areas. Indeed, the LPIS are produced at national scale each year. Results have however to be produced on numerous and diverse areas. Several im- 
Table 3. Effect on temporal structure on Class Metrics

\begin{tabular}{c|ccc}
\hline Class & F-score & Recall & Precision \\
\hline Corn & \multicolumn{3}{|c}{ Unstructured } \\
\hline Barley & 0.941 & 0.929 & 0.953 \\
Other cereals & 0.898 & 0.937 & 0.862 \\
Rape seed & 0.959 & 0.956 & 0.9378 \\
Protein & 0.949 & 0.975 & 0.944 \\
Fiber plants & 0.974 & 1 & 0.968 \\
Forage crops & 0 & 0.1 & 0 \\
Permanent grasslands & 0.868 & 0.814 & 0.930 \\
Fruit trees & 0.010 & 0.090 & 0 \\
Vegetables & 0.895 & 0.914 & 0.877 \\
\hline Class & \multicolumn{3}{|c}{ Structured } \\
\hline Corn & 0.878 & 0.831 & 0.935 \\
Barley & 0.816 & 0.785 & 0.849 \\
Other cereals & 0.954 & 0.941 & 0.968 \\
Rape seed & 0.969 & 0.985 & 0.954 \\
Protein & 0.953 & 0.967 & 0.939 \\
Fiber plants & 00 & 0.1 & 0 \\
Forage crops & 0.705 & 0.778 & 0.648 \\
Permanent grasslands & 0.955 & 0.943 & 0.967 \\
Fruit trees & 0.940 & 1 & 0.892 \\
Vegetables & 0.451 & 0.970 & 0.297 \\
\hline
\end{tabular}

provement of the method can be proposed. Greater order of the Markov chain could be tested in the future with the upcoming parcel-based versions of the LPIS. Other structures could also be modeled such as spatial relationship between parcels. The exhaustiveness and the few mistakes contained in the LPIS give valuable label information. The approach proposed is this paper will be tested in prototype of operational systems. In this article we only assessed the effet of temporal structure after 1-year of satellite observations. The objective would be to apply the method to partial time series to propose a pre-filled LPIS declaration for the farmers

\section{REFERENCES}

[1] European Commission, "Towards future copernicus service components in support to agriculture?," https://ec.europa.eu/jrc/sites/ jrcsh/files/Copernicus_concept_note_ agriculture.pdf, 2016.

[2] M. Immitzer, F. Vuolo, and C. Atzberger, "First experience with sentinel-2 data for crop and tree species classifications in central europe," Remote Sensing, vol. 8, no. 3, pp. 166, 2016.

[3] J. Inglada, M. Arias, B. Tardy, O. Hagolle, S. Valero, D. Morin, G. Dedieu, G. Sepulcre, S. Bontemps, P. De- fourny, et al., "Assessment of an operational system for crop type map production using high temporal and spatial resolution satellite optical imagery," Remote Sensing, vol. 7, no. 9, pp. 12356-12379, 2015.

[4] N. Kussul, G. Lemoine, F. J. Gallego, S. V. Skakun, M. Lavreniuk, and A. Y. Shelestov, "Parcel-based crop classification in ukraine using landsat-8 data and sentinel-1a data," IEEE Journal of Selected Topics in Applied Earth Observations and Remote Sensing, vol. 9, no. 6, pp. 2500-2508, 2016.

[5] GISAT, "Czech agriculture national demonstrator," http://www.esa-sen2agri.org/ wp-content/uploads/docs/CzechAgri\% 20Final\%20Report\%201.2.pdf, 2017.

[6] J. Dury, N. Schaller, F. Garcia, A. Reynaud, and J. E. Bergez, "Models to support cropping plan and crop rotation decisions. a review," Agronomy for sustainable development, vol. 32, no. 2, pp. 567-580, 2012.

[7] J. Osman, J. Inglada, and J.-F. Dejoux, "Assessment of a markov logic model of crop rotations for early crop mapping," Computers and Electronics in Agriculture, vol. 113, pp. 234-243, 2015.

[8] CNES, "Plateforme d'exploitation des produits sentinel. https://peps.cnes.fr/rocket/," 2017, Accessed on December 6, 2017.

[9] THEIA, "Pôle thématique surfaces continentales theia. https://theia.cnes.fr/," 2017, Accessed on December 6, 2017.

[10] J.-S. Lee, "Digital image enhancement and noise filtering by use of local statistics," IEEE transactions on pattern analysis and machine intelligence, vol. 2, no. 2, pp. 165-168, 1980.

[11] A. Veloso, S. Mermoz, A. Bouvet, T. Le Toan, M. Planells, J.-F. Dejoux, and E. Ceschia, "Understanding the temporal behavior of crops using sentinel-1 and sentinel-2-like data for agricultural applications," $R e$ mote Sensing of Environment, vol. 199, pp. 415-426, 2017. 